\title{
Estudio de la gestión del compromiso y cultura organizacional de FLACSO
}

\section{Rotation of Public Managers. Problem of management or solution of political trust?}

INFORMACIÓN DEL

\section{ARTÍCULO}

Fecha de recepción: 28 de Febrero de 2020.

Fecha de aceptación: 3 de Junio de 2020
1 Ingeniero en Administración del Talento Humano, Universidad UTEEcuador.

E-mail: richard.cuenca@ute.edu.ec

Código ORCID:

https://orcid.org/0000-0001-8552-7108

$\overline{2}$ Candidato a Doctor en Dirección de Empresas, Universidad de Valencia. Docente-investigador, UIDE-Ecuador. E-mail: helopezpa@uide.edu.ec Código ORCID:

https://orcid.org/0000-0003-0456-5271

Richard Xavier Cuenca Galarza ${ }^{1}$, Héctor Alejandro López Paredes ${ }^{2}$

\section{Resumen}

El propósito del estudio es analizar la cultura organizacional y el compromiso de los colaboradores de FLACSO, empleando como instrumento de investigación un cuestionario generado sobre las variables de interés, para a través de la técnica estadística de correlación identificar el tipo de cultura que predomina y el tipo de prácticas de gestión - compromiso que pueden fortalecerla, aportando al conocimiento en el campo con evidencia de los posibles caminos que deben tomar los profesionales de talento humano en el modelamiento de la cultura de una organización.

\section{Palabras Clave:}

Cultura Organizacional; Compromiso; Símbolos; Rituales; Lenguaje; Gestión Cultural.

\section{Clasificación JEL: M12, M14, M54.}

\begin{abstract}
The purpose of the study is to analyze the organizational culture and the commitment of the FLACSO collaborators, using as a research instrument a questionnaire generated on the variables of interest, in order to use the statistical correlation technique to identify the type of culture that prevails and the type of management practices - commitment that can strengthen it, providing knowledge in the field with evidence of the possible paths that human talent professionals should take in modeling the culture of an organization.
\end{abstract}

\section{Keywords:}

CITACIÓN: Cuenca Galarza, R.X., \& López Paredes, H.A.(2020). Estudio de la gestión del compromiso y cultura organizacional de FLACSO. Podium, 37, 43-56.

Organizational Culture; Commitment; Symbols; Rituals; Language; Cultural management.

JEL Classification: M12, M14, M54.

\section{ENLACE DOI:}

http://dx.doi.org/10.31095/podium.202 0.37 .4

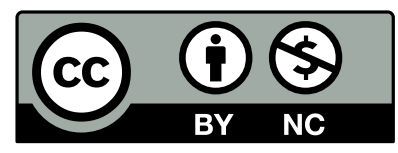




\section{Introducción}

Es necesario en toda organización identificar qué factores o elementos comprometen a los colaboradores para una correcta gestión del compromiso, permiten generar un apego de los colaboradores con la institución, y mayor afinidad hacia el cumplimiento de objetivos, además, de incrementar su deseo de permanecer en la organización. El compromiso organizacional involucra a la empresa y al trabajador, desarrollando vínculos para lograr una meta en conjunto, prevalece el sentido de pertenencia de la institución aceptando sus valores y objetivos (Amado, 2019).

De tal manera, si las personas están identificadas con la filosofía institucional (misión, visión, objetivos, la cultura, etc.), tienden a tener mayor adhesión con la institución. La presente investigación identifica cómo influye la cultura en el compromiso de los colaboradores, y para eso, se realizó una encuesta a los trabajadores de FLACSO, con la finalidad de medir la relación que existe entre las variables planteadas, de acuerdo a lo propuesto por Baez, Zayas, Velázquez y Lao (2019), el desconocimiento de la cultura organizacional limita la mejora del compromiso organizacional. Por otra parte, para Villacrés y López (2018), un factor que influye en el compromiso y que debe ser considerado para entender el clima de una organización es el tipo de liderazgo de sus principales líderes. Entendiendo que la cultura dicta el comportamiento de las personas en la institución y que estas están ligadas directamente a la estrategia de la empresa, por lo cual el tipo de liderazgo que se ejerce en la empresa influye en el comportamiento de los colaboradores, y, por ende en la cultura organizacional.

Los resultados arrojados por esta investigación, permitieron identificar cuáles son los elementos de la cultura de FLACSO que generan mayor compromiso en los colaboradores, además, de conocer cuál es la cultura que predomina en la institución, siguiendo lo mencionado por Barreno, López, y López (2018), "los procesos de investigación que faciliten al empresario nuevas herramientas para mejorar e innovar en su práctica diaria" (p. 66). En otras palabras, esta investigación busca aportar con una herramienta que permita a FLACSO conocer los elementos de su cultura que permitan mantener a sus colaboradores comprometidos con la institución.

\section{Revisión de literatura}

\section{Cultura Organizacional}

Según Morelos y Fontalvo (2014), "la cultura es a la organización, como la personalidad al individuo" (p.98), debido a que cada organización posee características propias que las diferencia de otras, por lo que Luján (2017), define la cultura organizacional como una herramienta que permite establecer las pautas que los colaboradores deben cumplir para conducirse en ésta, y siguiendo lo antes mencionado Gómez, (2019), "la cultura organizacional es un factor determinante para la toma de decisiones gerenciales y dada esta situación, cobra relevancia y 
utilidad contar con las herramientas necesarias para que este, se convierta en un factor positivo que conduzca a mejores decisiones" (p.7). Es decir, la cultura organizacional le da una identidad propia a la institución, debido a que cada organización posee una personalidad propia, a través de características que la distinguen del resto de organizaciones.

Según Ambrossi y Marconi (2017), la cultura organizacional es un conjunto de valores y comportamientos homogéneos, definidos por la organización y que deben ser vividos con intensidad por todos sus integrantes. En otras palabras, la cultura establece las normas de comportamiento de los individuos en la organización.

\section{Elementos de la Cultura Organizacional}

Serrate, Portuondo, Sánchez y Suárez (2014), manifiestan que los elementos de la cultura se encuentran respaldados por el estilo de liderazgo que predomina en la organización, mismo que facilitará la toma de decisiones. Según Villacrés y López (2018), los estilos de liderazgo permiten transmitir de manera positiva las directrices a los colaboradores respecto a lo que se debe hacer y cómo se lo debe hacer. Es decir, el estilo de liderazgo que posee la empresa permite ir modificando los comportamientos de las personas dentro de la institución, entendiendo que la cultura organización está estrechamente relacionada con el comportamiento de los colaboradores dentro de la empresa.

Asimismo Asencio, Fábregas, y Carmona (2019), señalan que los elementos de una cultura son inherentes a sus propias características, que generan una identidad a la institución, los que le permiten diferenciarse de otra institución, aun teniendo el mismo giro de negocio. De acuerdo con lo mencionado por varios autores los elementos de la cultura son los siguientes (ver Figura1):

\begin{tabular}{|c|c|c|}
\hline Autor & \multicolumn{2}{|c|}{ Elementos de la cultura organizacional } \\
\hline \multirow{3}{*}{$\begin{array}{c}\text { Hoistede } \\
\text { (1980) }\end{array}$} & \multirow{5}{*}{$\begin{array}{l}\text { - Valores } \\
\text { - Rituales } \\
\text { - Héroes } \\
\text { - Valores } \\
\text { - Mitos } \\
\text { - Símbolos }\end{array}$} & - Símbolos \\
\hline & & - Estructura y sistemas \\
\hline & & - Competencias \\
\hline \multirow[t]{2}{*}{ Boche (1984) } & & - Ritos \\
\hline & & - Héroes \\
\hline \multirow{2}{*}{ Shein (1992) } & \multirow{3}{*}{$\begin{array}{l}\text { - Artefactos y } \\
\text { creaciones }\end{array}$} & - Tejido cultural \\
\hline & & - Valores e ideología \\
\hline \multirow{4}{*}{$\begin{array}{c}\text { Gordon } \\
(1997, \\
\text { pp. } 474-476)\end{array}$} & & - Supuestos y premisas básicas \\
\hline & \multirow{3}{*}{$\begin{array}{l}\text { - El enunciado } \\
\text { de la misión } \\
\text { - Los héroes y } \\
\text { las heroínas }\end{array}$} & - Los mitos \\
\hline & & - Los rituales y las ceremonias \\
\hline & & - Las disposiciones físicas \\
\hline
\end{tabular}

Figura 1. Elementos de la cultura organizacional.

Fuente: Asencio et al., 2019.

\section{Tipos de cultura}

Para definir el tipo de cultura se parte de 2 dimensiones, que según Ruiz y Naranjo (2013), estas son: La primera dimensión, control versus flexibilidad, es decir, que la organización hace énfasis en el orden y el control, frente a las organizaciones que prefieren ser más discretas y dinámicas. La segunda dimensión tiene que ver con la orientación de la empresa, estas pueden ser tanto internas como externas. Para definir los tipos de cultura que existe, siguiendo el planteamiento de Hellriegel, Jackson y Slocum (2008), donde manifiesta que existen cuatro tipos de cultura organizacional (burocrática, la de clan, la emprendedora y la de mercado), que se caracterizan por las diferencias en 
el control formal (que va de estable a flexible) y el punto focal de la atención (que va de interno a externo).

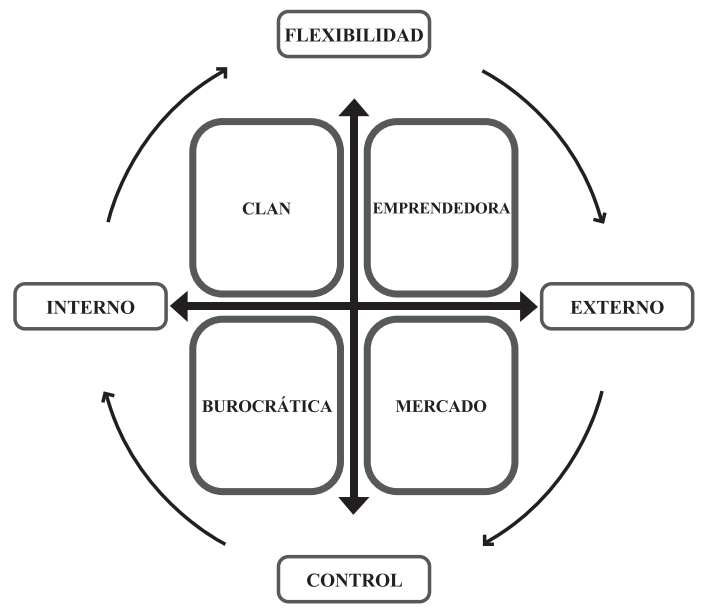

Figura 2. Tipos de cultura.

Fuente: Hellriegel et al., 2008, p. 602.

Los tipos de cultura son los siguientes:

- Burocrática: Da importancia al control y tiene una orientación interna, por otra parte, se valora lo formal, existe una estandarización en las reglas, los procedimientos, la operación, y la comunicación es de tipo vertical (Ritter, 2008).

- De clan: Da importancia a la flexibilidad y la orientación interna y, por ello, enfatiza el desarrollo humano, el trabajo en equipo, la participación de los trabajadores y el compromiso de todos con la organización (Ruiz, y Naranjo, 2013).

- Emprendedora: Las organizaciones son flexibles y tienen una orientación externa. Este tipo de cultura se caracteriza por asumir grandes riesgos, además, de impulsar los niveles de dinamismo, creatividad en sus colaboradores y su rápida adaptación a los cambios (Ritter, 2008).
- Mercado: Pone el énfasis en lo externo, pero requiere estabilidad y control para lograr mejoras en su productividad y competitividad (Ruiz y Naranjo, 2013).

\section{Compromiso}

Chiang, Gómez y Wackerling (2016) definen al compromiso organizacional como la fuerza con la que un individuo se siente vinculado a una organización. Asimismo Fierro, Martínez, Ortiz, y Martínez, (2018), manifiestan que el compromiso organizacional parte de un estado psicológico de las personas, mismo que puede influir en la continuidad de las personas en la organización, ya que sienten sus necesidades satisfechas o quizás porque sus objetivos se ven alineados con los de la organización. Siguiendo con lo antes mencionado Amado (2019), señala que "el compromiso organizacional involucra a la empresa y al trabajador, desarrollando vínculos para lograr una meta en conjunto, prevalece el sentido de pertenencia de la institución aceptando sus valores y objetivos" (p.14).

\section{Dimensiones del compromiso organizacional}

Según Allen y Meyer, (1990) y citado por varios autores, el compromiso es un estado psicológico, en donde: Los colaboradores se siente ligados emocionalmente a la organización, perciben costos asociados ante la posibilidad de dejar la organización o al sentir que tienen una obligación moral de permanecer en ella, se consideró tres 

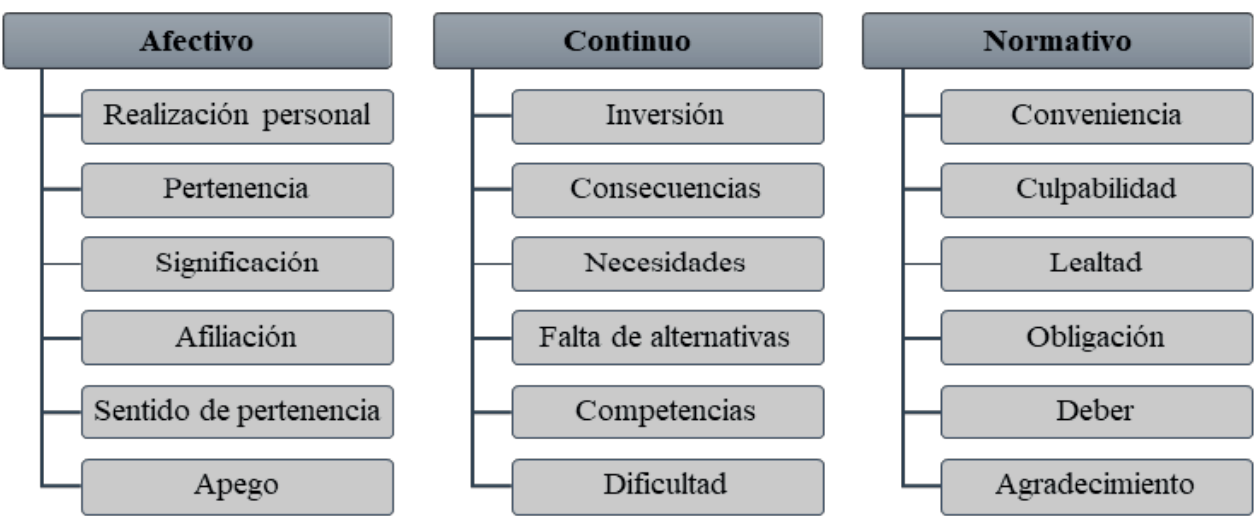

Figura 3. Dimensiones y variables del compromiso organizacional

Fuente: Báez Santana et al., 2019, p. 16.

componentes que pueden ser separables interrelacionados $y$ es a lo que se denomina Compromiso Afectivo, Compromiso Continuo y Compromiso Normativo (Báez Santana, Zayas Agüero, y Velázquez Zaldívar, 2019).

- Compromiso Afectivo (deseo): Los colaboradores generan lazos emocionales con la organización. Es decir, hay una fuerte atracción emocional, a la identificación y al involucramiento del empleado con la organización, los colaboradores desean permanecer en la organización (Fonseca, Cruz y Chacón, 2019).

- Compromiso de Continuidad (necesidad): El colaborador siente la necesidad de permanecer en la organización por lo que ve sus oportunidades reducidas fuera de la institución, y considera que al abandonarla perdería lo invertido (tiempo, dinero, esfuerzo), que según Báez Santana, et al. (2019) se relaciona con los costos asociados a dejar la organización.

- Compromiso Normativo (deber): Los colaboradores generan este tipo de compromiso con la organización, al sentirse en deuda con esta por los beneficios obtenidos. En otras palabras, sentimiento de obligación de permanecer en la organización por parte del empleado por todos los beneficios obtenidos (Ávila, 2015).

\section{Cómo generar compromiso en los colaboradores}

Según Jericó (2008), para generar compromiso se requiere del uso de herramientas que se pueden utilizar en diferentes fases y según donde se quiera incidir, entre ellas tenemos:

- Selección.

- Formación de los mandos intermedios.

- Difusión de la visión y valores de la organización.

- Adecuada definición del puesto de trabajo.

- Planes de carrera que permitan concretar expectativas futuras.

- Reconocimiento de los resultados.

- Estabilidad laboral.

- Sentimiento de pertenencia.

- Comunicación bidireccional.

- Entre otros. 
Influencia de la cultura organizacional en el compromiso de los colaboradores

Para Luján (2017) la cultura organizacional es uno de los pilares fundamentales para que una organización pueda tener éxito, debido a que esta le da una identidad que le hace única $y$ diferente al resto de organizaciones, de tal modo, que logra establecer un sentido de confianza y compromiso en los colaboradores, y a su vez los colaboradores valoran su trabajo, cumplen con sus funciones y se sienten orgullosos de pertenecer en ella. Es decir, al comprender y aceptar la cultura, los colaboradores se sienten identificados con la organización y es bastante probable que se alineen a los objetivos organizacionales con los personales, logrando así, que los colaboradores tengan un mejor desempeño y mayor productividad, con la finalidad de seguir formando parte de la organización por mucho más tiempo.

\section{Metodología}

Para el desarrollo del presente estudio se utilizó un método inductivodeductivo, por lo que, la investigación parte de la revisión bibliográfica y los resultados finales serán contrastados con la misma, que según lo expresado por Bernal (2010), define que "este método de inferencia se basa en la lógica $y$ estudia hechos particulares, aunque es deductivo en un sentido (parte de lo general a lo particular) e inductivo en sentido contrario (va de lo particular a lo general)" (p.60).
De la misma forma, se creó un instrumento de estudio, el mismo que partió de la revisión bibliográfica sobre las variables cultura organizacional y compromiso, mismas que fueron analizadas y relacionadas entre sí, con la finalidad de diseñar e implementar un instrumento (encuesta) que responda a la revisión de la información documental de las variables objeto de estudio, como son la cultura organizacional y el compromiso, y para segmentar la información se implementó los datos de identificación, mismo que será detallada en la Tabla 1.

Tabla 1.

Datos de identificación para la encuesta

\begin{tabular}{|c|c|}
\hline $\begin{array}{c}\text { Datos de } \\
\text { identificación }\end{array}$ & La Justificación \\
\hline Edad & $\begin{array}{l}\text { El segmentar las encuestas de } \\
\text { esta manera es para conocer } \\
\text { la percepción de cada grupo } \\
\text { generacional en cuanto a las } \\
\text { variables a investigar. }\end{array}$ \\
\hline Género & $\begin{array}{l}\text { Permite conocer la } \\
\text { percepción de las variables } \\
\text { compromiso y y cultura } \\
\text { organización por género. }\end{array}$ \\
\hline Discapacidad & $\begin{array}{l}\text { Conocer como las personas } \\
\text { con capacidades diferentes } \\
\text { perciben las variables } \\
\text { mencionadas en la presente } \\
\text { investigación. }\end{array}$ \\
\hline $\begin{array}{l}\text { Unidad a la que } \\
\text { pertenece }\end{array}$ & $\begin{array}{l}\text { Esta segmentación permite } \\
\text { tener una mejor percepción } \\
\text { de las variables de la } \\
\text { investigación en cuanto a los } \\
\text { grupos ocupacionales. }\end{array}$ \\
\hline $\begin{array}{l}\text { Tiempo en la } \\
\text { organización }\end{array}$ & $\begin{array}{llr}\text { Se puede conocer que } & \text { tan } \\
\text { comprometidas están } & \text { los } \\
\text { colaboradores con } & \text { la } \\
\text { organización } & \text { y } & \text { su } \\
\text { permanencia en la misma. }\end{array}$ \\
\hline
\end{tabular}

Fuente: Elaboración propia. 
La investigación tiene una modalidad de campo, razón por la que las encuestas se realizaron en las instalaciones de FLACSO Ecuador, donde la muestra fue seleccionada a través de las técnicas de muestreo aleatorio simple y por conveniencia, después de haber concluido con el levantamiento de 127 encuestas, la base de datos se procesó en el programa IBM SPSS Statistics 25, y se realizó un estudio estadístico de tipo descriptivo y correlacional.

El estudio correlacional buscó determinar el grado de asociación de las variables de estudio, que según Revelo (2017) "establece que existe una relación altamente significativa entre las variables" (p.300), en consecuencia de acuerdo al nivel de asociación entre las variables de estudio, se puede elaborar la propuesta que permite identificar los elementos de la cultura que generan compromiso en los colaboradores de FLACSO.

\section{Resultados}

Una vez aplicadas las encuestas de acuerdo a la muestra seleccionada, se procedió a realizar su respectiva tabulación y el análisis descriptivo $\mathrm{y}$ correlacional de los resultados.

En la Figura 4, se puede observar que el $70 \%$ de los hombres se sienten identificados con los rasgos culturales de la institución, mientras que en las mujeres existe un descenso en la identificación con la cultura en un 57\%, contrastando en este caso lo manifestado por Sánchez-Apellániz (1997), las mujeres se adaptan mejor a la cultura que los hombres. Por otra parte en la Figura 5, se puede observar que en FLACSO no existe una diferencia muy amplia en relación al conocimiento de los objetivos tanto en hombres $(63 \%)$ como mujeres (67\%), así como lo menciona Aldana (2013), entre hombres y mujeres no existe una diferencia en el conocimiento y cumplimiento de objetivos.

Elementos de la cultura

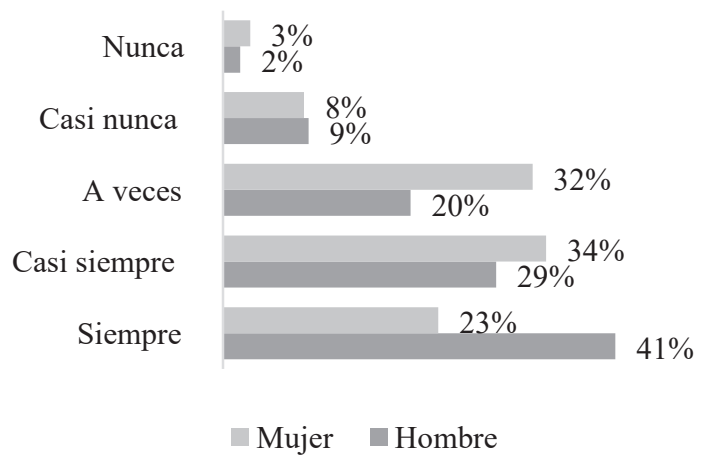

Figura 4. Existen rasgos culturales (creencias, valores, lenguajes, etc.) que identifique a las personas que forman parte de FLACSO.

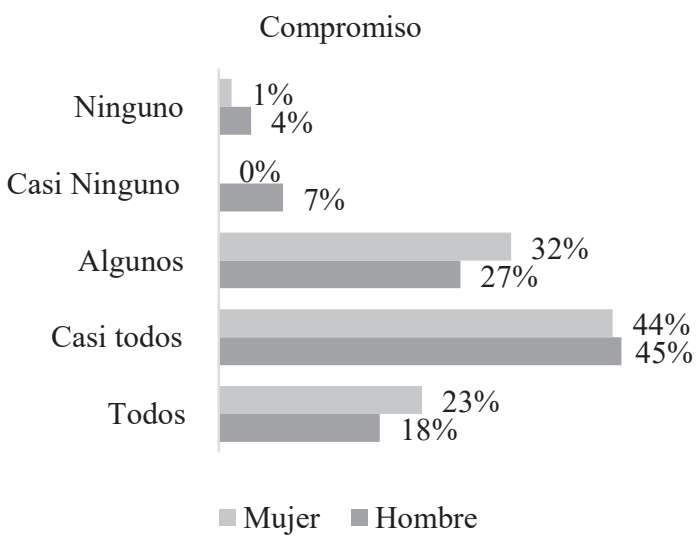

Figura 5. Conoce los objetivos estratégicos de FLACSO.

Asimismo, en la Figura 6, se evidenció que los colaboradores de FLACSO, tanto hombres como mujeres tienen un limitado conocimiento de los valores institucionales, siendo el $67 \%$ de los hombres que conocen los valores contra un $64 \%$ de mujeres, siguiendo lo mencionado por Ávila (2015), que tanto 
hombres como mujeres tienen valores similares en cuanto a su compromiso.

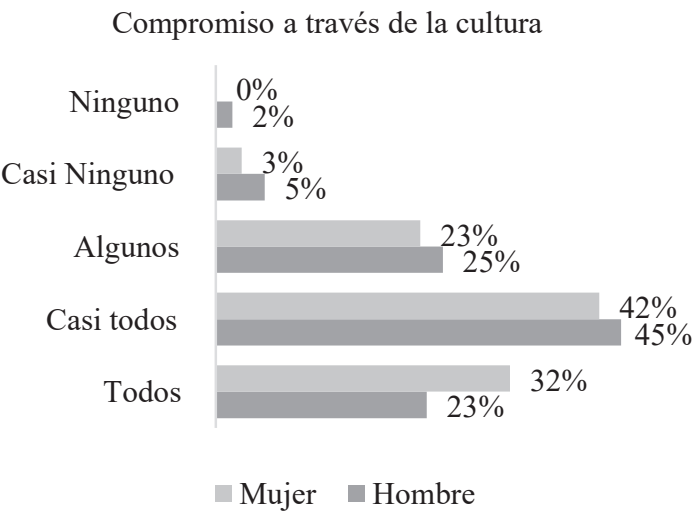

Figura 6. Conoce los valores institucionales de FLACSO.

\section{Tipos de cultura}

Los elementos identificados encasillan rasgos culturales burocrático en un $89 \%$ de las personas encuestadas, quienes consideran que deben regirse a normas y procedimientos ya establecidos para el cumplimiento de sus actividades (ver Figura 7), como de mercado ya que el $87 \%$ de las personas encuestadas, considera que FLACSO está orientado más al cumplimiento de resultados (ver Figura 8). De acuerdo a lo mencionado con anterioridad, la cultura que más prevalece es la cultura de tipo burocrática.

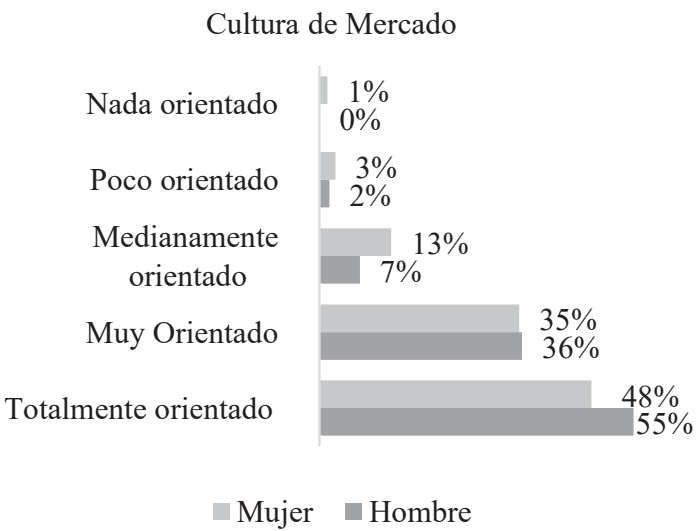

Figura 7. En el desempeño diario de sus actividades, con qué frecuencia debe regirse a normas y procedimientos ya establecidos.

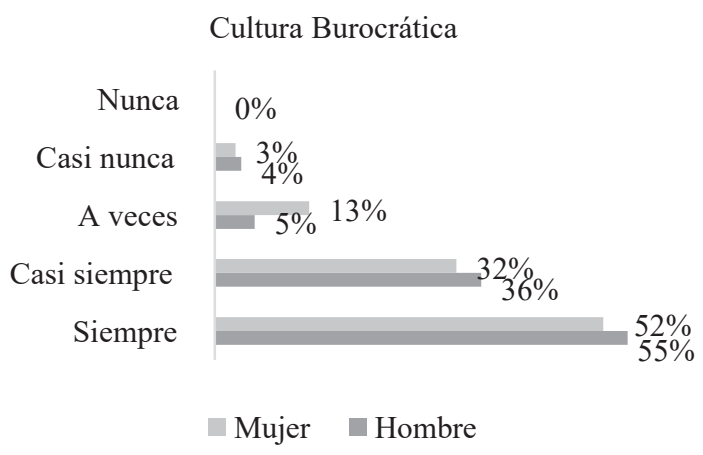

Figura 8. El trabajo que usted desempeña está orientado al cumplimiento de los objetivos institucionales.

De acuerdo a la Figura 9, los hombres en un $73 \%$ consideran que en FLACSO se promueve el trabajo en equipo, frente a un $62 \%$ de las mujeres que opinan lo mismo, contrastando lo mencionado por García Solarte, García Pérez De Lema y Madrid Guijarro (2018), las mujeres están más orientadas al trabajo en equipo que los hombres.

$$
\text { Cultura de Clan }
$$

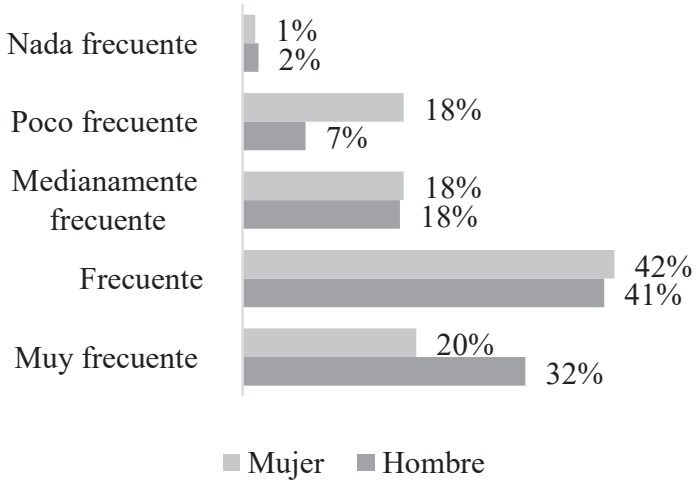

Figura 9. Con qué frecuencia FLACSO promueve el trabajo en equipo de sus colaboradores.

Asimismo, en la Figura 10 se puede observar que en FLACSO se desarrolla medianamente los niveles de creatividad de los colaboradores, considerando los resultados manifestados, el $69 \%$ de los hombres afirman que la institución desarrolla sus niveles de iniciativa y creatividad en las actividades que desempeñan, frente a un $48 \%$ de las 
mujeres que realizan la misma afirmación, por otra parte, se evidencia que si las mujeres asumieran más riesgos, salieran de su zona de confort, y tuvieran una mejor motivación para proponer, lograrían de manera notable incrementar la percepción de que el predominio es de los varones, sin embargo, García Solarte et al. (2018), manifiestan que las mujeres asumen más riesgos que los hombres, razón por la cual desarrollan los niveles de iniciativa y creatividad en mayor medida que los hombres.

\section{Cultura Emprendedora}

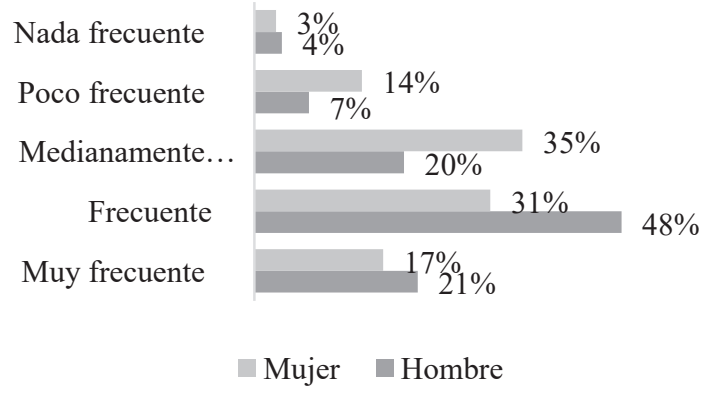

Figura 10. Con qué frecuencia FLACSO desarrolla los niveles de creatividad y fomenta al emprendimiento de una actividad nueva o por voluntad propia en su entorno laboral.

Compromiso normativo (deber)

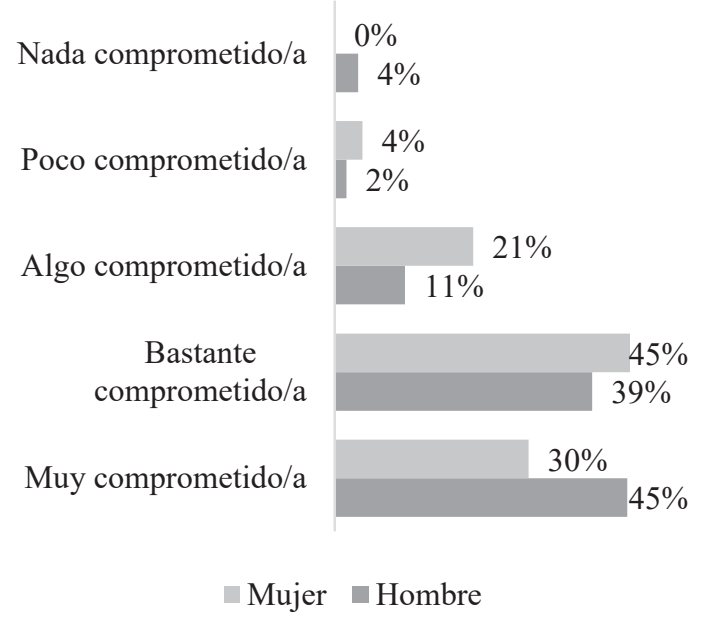

Figura 11. Se siente usted comprometido/a con FLACSO por los beneficios económicos ofrecidos por la institución.

\section{Dimensiones del compromiso identificado en el estudio}

De acuerdo a las dimensiones de compromiso que tienen los colaboradores con FLACSO, se identifica que existe un compromiso normativo (deber), puesto que los colaboradores de FLACSO se sienten más comprometidos por los beneficios otorgados por la institución, tanto económicos en un $80 \%$, como no económicos en un $82 \%$ (ver Figuras 11, 12), y un compromiso afectivo (deseo), en donde un $89 \%$ se sienten orgullosos de trabajar en FLACSO y un $74 \%$ que sienten los problemas de FLACSO como propios (ver Figuras 13, 14), donde prevalece el compromiso afectivo por una mínima diferencia sobre el compromiso normativo.

En la Figura 15, los resultados del presente estudio muestran que en los colaboradores de la institución predomina un sentido de permanencia, más en los hombres que en las mujeres,

Compromiso normativo (deber)

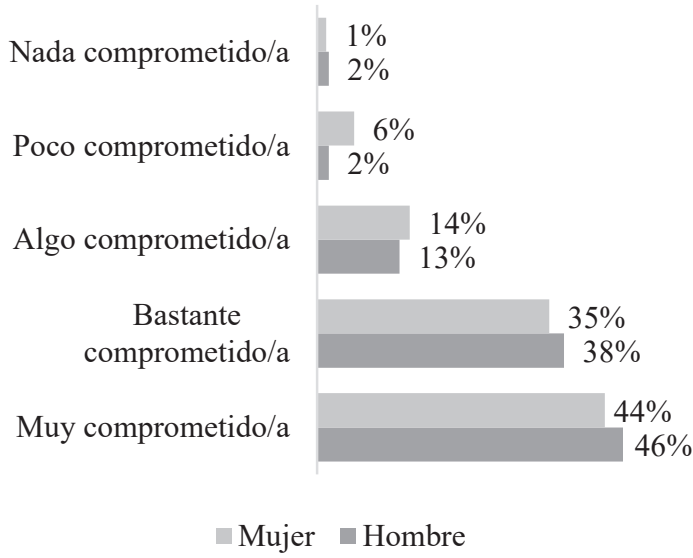

Figura 12. Se siente usted comprometido/a con FLACSO por los beneficios no económicos (emocionales como vacaciones adicionales, opción de estudios sin costo en FLACSO o flexibilidad en los horarios) ofrecidos por la institución. 
Compromiso afectivo (deseo)

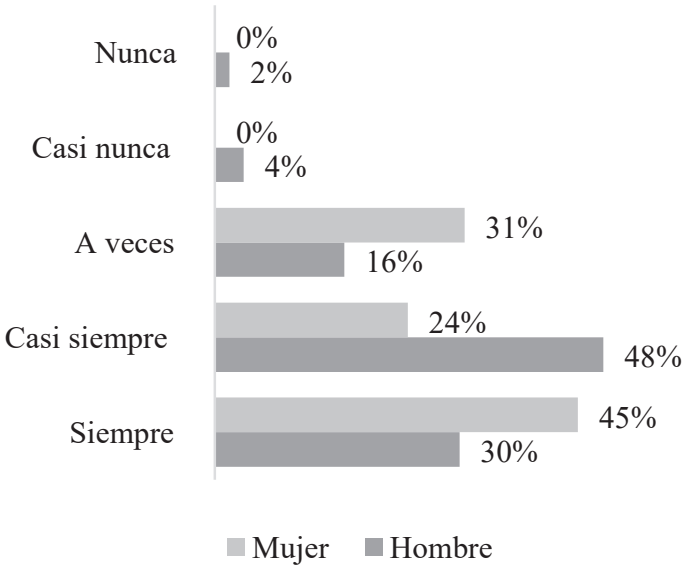

Figura 13. Qué tan orgulloso/a se siente de trabajar en FLACSO.

misma que se evidencia al observar la Figura 11, donde predomina la opción intermedia, y es más fuerte en las mujeres que en los hombres, que de acuerdo a Céspedes (2017), "El compromiso de continuidad es más predominante en varones con más años de trabajo a diferencia de las damas" (p.43).

\section{Correlación}

El compromiso de los colaboradores se encuentra en la identificación de las personas con los valores institucionales, con una relación moderada de 0,441 (ver Tabla 2) con el factor cumplimiento de los objetivos institucionales, es decir, si los colaboradores no se sienten identificados es difícil que puedan cumplir con los resultados organizacionales, que respalda lo expresado por Rocha y Böhrt (2004), las personas comprometidas buscan cumplir los objetivos institucionales, sintiéndose miembros activos de la empresa y mostrando lo mejor de ella. En el factor Compromiso normativo (deber) tiene una
Compromiso afectivo (deseo)

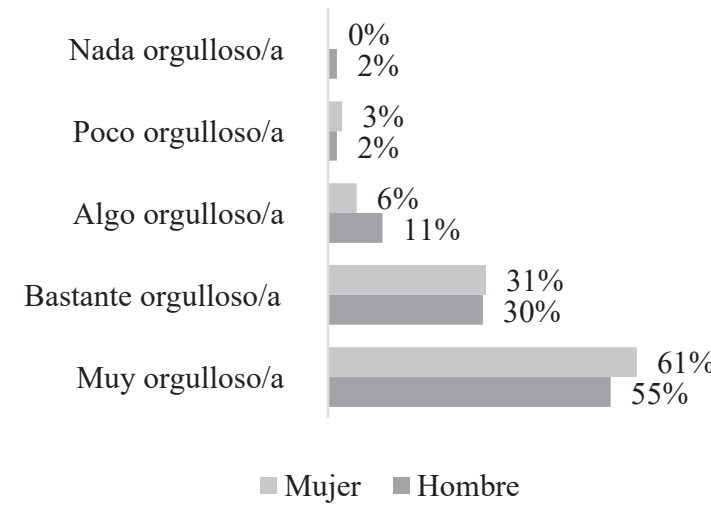

Figura 14. Siente los problemas de FLACSO como propios.

Compromiso de continuación (necesidad)

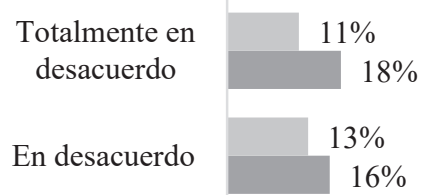

Ni de acuerdo, ni en desacuerdo

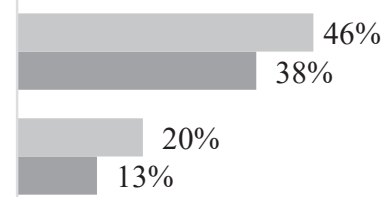

Totalmente de acuerdo

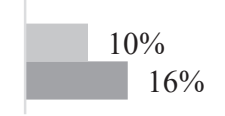

$$
\square \text { Mujer } \square \text { Hombre }
$$

Figura 15. En caso de presentarse una mejor oportunidad laboral, consideraría abandonar su empleo actual en FLACSO.

Tabla 2.

Correlaciones significativas de cultura y compromiso organizacional

\begin{tabular}{|c|c|c|}
\hline & \multicolumn{2}{|c|}{$\begin{array}{l}\text { Cumplimiento de } \\
\text { resultados }\end{array}$} \\
\hline \multirow{3}{*}{$\begin{array}{l}\text { Identificación } \\
\text { de los } \\
\text { colaboradores }\end{array}$} & Correlación de Pearson &, $441^{* *}$ \\
\hline & Sig. (bilateral) & 0,000 \\
\hline & $\mathrm{N}$ & 127 \\
\hline \multirow{3}{*}{$\begin{array}{l}\text { Compromiso } \\
\text { normativo }\end{array}$} & Correlación de Pearson & $471^{* *}$ \\
\hline & Sig. (bilateral) & 0,000 \\
\hline & $\mathrm{N}$ & 127 \\
\hline
\end{tabular}

Nota: Significancia de 0 a 0,05 .

Fuente: Elaboración propia. 
relación de 0,471 con el factor de cumplimiento de resultados, en el que se considera que los beneficios no económicos como el salario emocional permiten que los colaboradores estén más orientados al cumplimiento de los objetivos institucionales, de acuerdo a lo manifestado por Amado, (2019), el reconocimiento es una necesidad psicológica que permite obtener resultados alentadores orientados al logro de resultados.

Según Hernández Sampieri, Fernández Collado, y Baptista Lucio (2010), "los estudios descriptivos por lo general son la base de las investigaciones correlacionales, las cuales a su vez proporcionan información para llevar a cabo estudios explicativos que generan un sentido de entendimiento y son altamente estructurados" (p. 78). Posterior a la aplicación de las encuestas a los colaboradores, el procesamiento de la información se realizó a través de un análisis descriptivo y correlacional. En efecto, en la presente investigación se realizó un análisis descriptivo, para tener una visión más clara y detallada de los resultados obtenidos de la misma, permitiendo así una mejor interpretación.

Se realizó un análisis de tipo correlacional para determinar el grado de asociación que tienen los variables compromiso y cultura organizacional, que permitirá generar la propuesta con las asociaciones más fuertes. De esta manera Báez y Jongitud,(2014) mencionan que las variables son más fuertes en valores cercanos a $+/-1$, por el contrario una relación se hace más débil o nula mientras más se acerque a cero (ver Tabla 3), complementando lo antes mencionado Murray y Larry (2009), afirman que para que una correlación sea válida debe tener un nivel de significancia de 0.05 y 0.01 , puesto que los valores mayores a 0.05 no se consideran estadísticamente significativos.

Tabla 3.

Interpretación de resultados de la correlaciónsectoriales

\begin{tabular}{|c|l|}
\hline $\begin{array}{c}\text { Valor de la } \\
\text { correlación }\end{array}$ & \multicolumn{1}{|c|}{ Significado } \\
\hline-1 & $\begin{array}{l}\text { Correlación negativa grande y } \\
\text { perfecta }\end{array}$ \\
\hline$-0,9$ a $-0,99$ & Correlación negativa muy alta \\
\hline$-0,7$ a $-0,89$ & Correlación negativa alta \\
\hline$-0,4$ a $-0,69$ & Correlación negativa moderada \\
\hline$-0,2$ a $-0,39$ & Correlación negativa baja \\
\hline$-0,01$ a $-0,19$ & Correlación negativa muy baja \\
\hline 0 & Correlación nula \\
\hline 0,01 a 0,19 & Correlación positiva muy baja \\
\hline 0,2 a 0,39 & Correlación positiva baja \\
\hline 0,4 a 0,69 & Correlación positiva moderada \\
\hline 0,7 a 0,89 & Correlación positiva alta \\
\hline 0,9 a 0,99 & Correlación positiva muy alta \\
\hline 1 & $\begin{array}{l}\text { Correlación positiva grande y } \\
\text { perfecta }\end{array}$ \\
\hline
\end{tabular}

Fuente: Báez \& Jongitud, 2014, p.138

\section{Conclusiones}

La cultura de FLACSO a partir de la investigación muestra que la cultura burocrática mantiene el predominio sobre los otros tipos de cultura, puesto que se tiene como fin el cumplimiento de resultados en base a procesos sistemáticos preestablecidos, según afirma Toca y Carrillo (2009), basa su organización en la jerarquía de la funciones, además de la comunicación en línea recta de tipo ascendente, es decir formal; seguido de una cultura de mercado que para Hellriegel et al. (2008), es aquella cultura que rige sus valores y normas en el cumplimiento de metas con criterio en el mercado y financiero. Los 
resultados arrojados muestran que los colaboradores no reconocen la cultura de la institución, siendo estos los símbolos, historias, ritos o lenguajes los elementos medianamente desconocidos por los mismos, que según Luján (2017), la identidad de los colaboradores generados a partir de la cultura institucional, permite comprometer a la organización y alcanzar sus objetivos.

De acuerdo a los resultados de la investigación, se concluyó que se conoce algunos o ninguno de los objetivos, el $38 \%$ los hombres, y las mujeres un 33\%, frente al conocimiento de alguno $\mathrm{o}$ ninguno de los valores institucionales, un $32 \%$ de hombres y un $26 \%$ en mujeres del total de personas encuestadas laboran en FLACSO, la identidad institucional de acuerdo a su filosofía es relativamente baja, basados en el conocimiento de los valores institucionales, objetivos, políticas, entre otros, asimismo Morelos y Fontalvo (2014), señalan que la filosofía institucional determina las metas, objetivos, valores, y acciones que se deben ejecutar para el logro de los objetivos, además se evidencia en los colaboradores el sentido de pertenencia, además de su sentimiento de participación, elevando su compromiso afectivo con la institución, siguiendo lo mencionado por Chiang, Núñez, Martín, y Salazar (2010), "el compromiso afectivo se refiere a los lazos emocionales que las personas forjan con la organización al percibir la satisfacción de sus necesidades y expectativas; por ende, disfrutan de su permanencia en la organización" (p.91), de igual forma se puede apreciar en los colaboradores el compromiso de tipo normativo, en el que se identifican por los beneficios no económicos de la institución, que según Ávila (2015), "el compromiso normativo es el sentimiento de obligación de permanecer en la organización por parte del empleado por todos los beneficios obtenidos" (p.8).

En criterio al análisis de resultados y la revisión bibliográfica, se determinó que las relaciones del factor cumplimento de resultados son los factores que más influyen en la investigación; el factor identificación de los colaboradores en un $44 \%$, mientras que en un $47 \%$ el factor compromiso normativo (deber), los mismos en que basará la propuesta.

Con base a la cultura predominante de la institución de encaminar las actividades, agilizar la comunicación entre colaboradores en la que se comparta la información de la cultura institucional y los elementos que la conforman, en la cual se vea reflejado a profundidad la historia de FLACSO. Para corroborar la eficiencia de las medidas adoptadas es necesario realizar encuestas en la que se refleje los resultados de la identidad de los colaboradores en relación a la cultura institucional.

Con el objetivo de aumentar el compromiso y fidelidad en los colaboradores es necesario implementar medidas que permitan generar un mayor apego con la institución, como reconocimientos no económicos, puesto que es necesario elevar el sentimiento de pertenencia y de importancia dentro de la institución, además, de brindar a cada 
sujeto la oportunidad de superarse dentro de su entorno laboral a pesar de su limitada rotación. Además, de permitir una mayor formación y capacitación en cargos que se considere importantes en FLACSO. Es necesario de igual manera que estas medidas permitan que los colaboradores conozcan y se identifiquen con la filosofía institucional.

Elaborar un plan de identificación institucional que permita a los colaboradores identificarse con la cultura de FLACSO, además, establecer un plan de reconocimiento que permitirá hacer más fuerte el compromiso de tipo afectivo y los colaboradores deseen permanecer en la institución.

\section{Referencias}

Aldana, J. (2013). Compromiso laboral de los trabajadores del área central de una institución bancaria que opera en la ciudad de guatemala, según sexo. Tesis de grado, Universidad Rafael Landívar.

Allen, N. J., y Meyer, J. P. (1990). The measurement and antecedents of affective, continuance and normative commitment to the organization. Journal of Occupational Psychology, 63(1), 1-18.

Amado, E. (2019). Motivación laboral y Compromiso organizacional en empleados de una empresa privada del rubro salud en Lima. Tesis de grado, Universidad Nacional Federico Villarroel.

Ambrossi, D., y Marconi, L. (2017). Diagnóstico de la Cultura Organizacional en el Banco de Loja. PODIUM Edición Especial, 7-27.

Sánchez-Apellániz, M. S. (1997).Culturas y estilos directivos en hombres y mujeres: Un análisis empírico. Revista europea de dirección y economía de la empresa, 6(1), 57-74.

Asencio, L., Fábregas, C., y Carmona, C. (2019). Cultura organizacional y el intraemprendimiento académico en la Universidad de Guayaquil-Ecuador. Desarrollo Gerencial, 11(1), 79-103.

Ávila, C. E. (2015). Plan de compromiso laboral (engagement) en el área comercial de laboratorios Life 2015. Tesis de grado, Universidad UTE.

Báez, J. F., \& Jongitud, J. (2014). La influencia de la corrupción sobre el derecho a una educación de calidad. Un estudio de correlación. Revista ProlegómenosDerechos y Valores, 17(33), 123-142.

Báez Santana, R. A., Zayas Agüero, P. M., Velázquez Zaldívar, R., \& Lao León, Y. O. (2019). Modelo conceptual del compromiso organizacional en empresas cubanas. Ingeniería Industrial, 40(1), 14-23.

Barreno, L., López, H., y López, M. (2018). Relación investigación, innovación: el desarrollo de las empresas ecuatorianas con las universidades. PODIUM, 33, 55-68. https://doi.org/10.31095/podium.2018.33.6

Bernal, C. (2010). Metodología de la investigación (3a ed.). Pearson Educación: Colombia.

Céspedes Correa, F. (2017). Comunicación organizacional y compromiso organizacional en docentes de instituciones educativas públicas del distrito de San Martín de Porres, 2017. Tesis de grado, Universidad César Vallejo.

Chiang, Margarita, Núñez, A., Martín, M. J., \& Salazar, M. (2010). Compromiso del Trabajador hacia su Organización y la relación con el Clima Organizacional: Un Análisis de Género y Edad. Panorama Socioeconómico, 28(40), 90-100.

Chiang, M., Gómez, N., y Wackerling, L. (2016). 
Compromiso Organizacional del Funcionario Municipal Rural de la Provincia de Nuble, Chile. Ciencia \& Trabajo, 18(56), 134-138.

Fierro, E., Martínez, M., Ortiz, F. A., y Martínez, J. (2018). La Gestión Pública Colaborativa y el Compromiso Organizacional. Organizaciones del sector público del Estado de México. RECAI,7(20), 1-13.

Fonseca, J. M. G., Cruz, C. E., y Chacón, L. (2019). Validación del instrumento de compromiso organizacional en México: evidencias de validez de constructo, criterio y confiabilidad. Revista de Psicología, 37(1), 7-29. https://doi.org/10.18800/ psico.201901.001

García Solarte, M., García Pérez De Lema, D., y Madrid Guijarro, A. (2018). El género de la gerencia y la cultura organizacional de la pyme en la región de Murcia. Revista Venezolana de Gerencia,23(82).

Gómez, J. L. (2019). La cultura organizacional y la toma de decisiones gerenciales. Tesis de postgrado, Universidad Militar Nueva Granada.

Hellriegel, D., Jackson, S. E., y Slocum, J. W. (2008). Administración: Un enfoque basado en competencias (11th ed.). México: Cengage Learning.

Hernández Sampieri, R., Fernández Collado, C., y Baptista Lucio, M. (2010). Metodología de la investigación (6a ed.). México: Mc Graw Hill Education.

Jericó, P. (2008). Gestión del compromiso. Factor Humano.

Luján, G. L. (2017). Cultura organizacional y compromiso laboral en el Hospital I La Esperanza -Essalud La Libertad. Tesis de grado, Universidad César Vallejo. los factores determinantes de la cultura organizacional en el ambiente empresarial. Entramado, 10(1), 96-105.

Murray, S., y Larry, S. (2009). Estadística (4ta. ed.). México: Mc Graw Hill.

Revelo, J. E. (2017). Modelo de integración de la competencia digital docente en la enseñanza de la Matemática en la Universidad Tecnológica Equinoccial. Tesis doctoral, Universidad de Extremadura.

Ritter, M. (2008). Cultura Organizacional: gestión y comunicación (1a ed.). Buenos Aires: La Crujía ediciones.

Rocha, M., y Böhrt, M. (2004). Tres dimensiones del compromiso organizacional: identificación, membresía y lealtad. Ayaju, 2(1).

Ruiz, Y. B., y Naranjo, J. C. (2013). La investigación sobre cultura organizacional en Colombia: una mirada desde la difusión en revistas científicas. Diversitas: Perspectivas en Psicología, 8(2), 285-307.

Serrate, A., Portuondo, Á. L., Sánchez, N., y Suárez, R. (2014). Evaluación de la cultura organizacional y su incidencia en la efectividad grupal. Ingeniería Industrial, 35 (1), 2-12.

Toca, C., y Carrillo, J. (2009). Asuntos teóricos y metodológicos de la cultura organizacional. Civilizar, 9(17), 117-136.

Villacrés, J., y López, H. (2018). Incidencia del tipo de liderazgo en el clima laboral. Caso: área de cajas de las agencias de Quito de una entidad financiera. Economía y Negocios, 9(1). https://doi.org/10.29019/eyn.v9i1.429 